\title{
Qualité De La Formation Et \\ Des Enseignements Dispensés Par Les Instituteurs Ordinaires Et Les Instituteurs Adjoints : Points De Vue Des Professeurs Des Cafop Et Des Conseillers Pédagogiques Des Secteurs De L'enseignement Primaire
}

\author{
Coulibaly Abdoulaye \\ Doctorant en Sciences Sociales de Développement du Capital Humain, \\ Université Félix Houphouët-Boigny (ISAD), Abidjan, Côte d'Ivoire
}

Doi:10.19044/esj.2018.v14n34p178 URL:http://dx.doi.org/10.19044/esj.2018.v14n34p178

\begin{abstract}
Good teacher training is one of the keys to ensuring that students receive good quality of teaching. Due to economic reasons in Ivory Coast, there has been a massive recruitment of teachers with a lower quality of educational training compared to what was previously considered. As a result, several questions about the quality of the lessons have emerged. However, this paper focuses on collecting the points of view of the CAPT teachers (Center for Animation and Pedagogical Training) and the educational advisers to the primary educational sectors in regards to the quality of training and the teaching provided by assistant teachers and ordinary teachers. In Ivory Coast, it is the CAPTs who trains the teachers who teaches in primary schools. The answers collected and analyzed allow us to conclude that although these two types of teachers have almost identical training, the level of the quality of the teaching provided by the assistant teachers is lower than that of the ordinary teachers. The general training acquired before becoming part of this body of teachers appears as the main reason for this difference.
\end{abstract}

Keywords: Training, teaching, teachers, teachers of CAFOP, educational advisers of sectors

Résumé

La bonne formation des enseignants est l'un des gages pour que les élèves aient des enseignements de bonne qualité. Pour des raisons économiques, en Côte d'Ivoire, l'on est passé à un recrutement massif d'instituteurs ayant d'un diplôme inférieur à celui qui était utilisé auparavant. 
Pour cette raison, on se pose des questions concernant la qualité des enseignements qui en ressortent. Ainsi, cette étude vise à recueillir les points de vue des professeurs des CAFOP (Centre d'Animation et de Formation Pédagogique) et des conseillers pédagogiques de secteurs de l'enseignement primaire sur la qualité de la formation et des enseignements dispensés par les instituteurs adjoints et les instituteurs ordinaires. En Côte d'Ivoire, ce sont les CAFOP qui forment les instituteurs qui interviennent dans l'enseignement primaire. Les réponses recueillies et analysées permettent de conclure que bien que ces deux types d'instituteurs aient une formation quasiment identique, le niveau de qualité des enseignements dispensés par les instituteurs adjoints est inférieur à celui des instituteurs ordinaires. La formation générale acquise avant de s'incorporer à ce corps d'enseignants apparaît comme la raison principale expliquant cet écart.

Mots-Clés: Formation, enseignements, instituteurs, professeurs des CAFOP, conseillers pédagogiques de secteurs

\subsection{INTRODUCTION}

De plus en plus, l'on parle de la baisse du niveau de l'enseignement en Côte d'Ivoire. Certains vont même jusqu'à dire que le système éducatif ivoirien est en déliquescence. À cet égard, nous avons cherché à connaître d'où venait le mal. Nous nous sommes dit qu'il fallait analyser le mal de façon scientifique pour en ressortir des recommandations qui pourraient faire fonctionner mieux le système éducatif. Les causes de ces dysfonctionnements peuvent être nombreuses: l'insuffisance d'infrastructures scolaires, une pédagogie mauvaise, inadaptée ou mal appliquée, des problèmes dus aux acteurs...

Assurer la qualité de la formation reste complexe au regard de son caractère multidimensionnel (UNESCO, 2002). La réussite d'une telle action requiert de mettre en œuvre plusieurs fonctions et activités (enseignement, programme académique, ressources humaines, équipement) (Niasse, 2009).

Bien que la qualité de la formation soit une exigence, elle reste un but que l'on recherche (Woodhouse, 1999). La Banque Mondiale (2003) préconise l'adoption de nouveaux mécanismes qui mettent l'accent sur la corrélation entre financement et performance au niveau des établissements de la formation. À ce niveau de la question, se pose le problème de la mesure de la qualité de la formation. Woodhouse (1999) soutient qu'on ne peut apprécier la qualité d'un bien ou d'un service que si l'on procède à son évaluation. Il précise que dans le cadre de la formation, l'évaluation permet de vérifier si un établissement donne à ses apprenants les qualités nécessaires pour accéder à un emploi dans un domaine spécifique. 
La formation des enseignants, aujourd'hui est l'un des gages pour avoir des enseignements de bonne qualité. La qualité est le degré avec lequel, pour un élève donné, la présentation, l'explication, l'ordonnancement des éléments de la tâche à apprendre s'approchent d'un optimum (Bloom, 1969). Mais essentiellement pour des raisons économiques, l'on est passé à un recrutement massif d'instituteurs possédant un diplôme de niveau inférieur à celui auquel cela était fait naguère. Des questions sont donc posées concernant la qualité des enseignements qui en ressortent. C'est ce dernier point, celui des acteurs, ce qui nous a intéressés, plus précisément les enseignants.

Cette formation des enseignants, aujourd'hui plus qu'hier, est considérée comme un levier direct pour agir sur les résultats de l'école. Face à une société en transformation rapide, elle constitue une priorité et fait l'objet de multiples réformes à l'échelle nationale. Hormis quelques enseignants bénévoles recrutés de façon locale et n'émargeant pas au budget de l'État, l'on dénombre deux types d'instituteurs dans l'enseignement primaire en Côte d'Ivoire: les instituteurs ordinaires et les instituteurs adjoints. "Le recrutement et la formation d'instituteurs adjoints visent à faire baisser les charges afin qu'elles soient soutenables pour les finances publiques, tout en étant proches de la moyenne constatée dans les pays africains ayant atteint la scolarité universelle ou en passe de l'atteindre » (Koffi, 2012, p.62).

À partir de 1966, les premiers centres d'animation et de formation pédagogique (CAFOP) s'ouvrent en Côte d'Ivoire et sont appelés CAFOP. Ils étaient chargés de former les instituteurs adjoints recrutés à partir du niveau BEPC ou BE. L'École Normale d'Instituteurs (ENI) ouvre ses portes en 1969 à Bouaké, et elle forme des instituteurs ordinaires stagiaires en 2 ans qui sont titularisés à la fin d'un stage pratique d'un an. L'admission se fait sur concours ouvert aux instituteurs adjoints titulaires du Certificat Élémentaire d'Aptitude Pédagogique (CEAP). À partir de 1983, les CAFOP recrutent uniquement au baccalauréat, le niveau 1 étant supprimé. La formation y dure un an et elle est ensuite portée à 2 ans. À partir de 1993, la formation dure 3 ans dont deux années de formation théorique au CAFOP et une année de formation pratique sur le terrain sanctionnée par l'examen pratique du Certificat Élémentaire d'Aptitude Pédagogique (CEAP). Depuis l'année scolaire 2000-2001, la formation a été réduite à une année. Après cette année, il y a eu le recrutement de plusieurs promotions d'instituteurs avec le BEPC jusqu'à son arrêt en 2011. Contre toute attente, le recrutement à partir du BEPC a été repris à la rentrée 2017-2018, et les instituteurs adjoints devront ainsi faire une année de formation théorique au CAFOP et deux années de formation pratique sur le terrain sanctionnées par l'examen du CEAP.

L'enseignant est un professionnel de l'apprentissage, de la gestion des conditions d'apprentissage et de régulation interactive en classe (Fauré, 2008). Son action est nécessairement appuyée sur une préparation dans laquelle il 
mobilise des connaissances professionnelles. Pour que cette préparation soit efficace, il faudrait que l'enseignant ait une formation solide à la base, et elle commence bien avant qu'il n'intègre le centre de formation.

L'écart est grand entre la classe de 3è où l'on présente le BEPC et la classe de terminale où l'on présente le baccalauréat. Nous avons trois années scolaires entre ces deux niveaux, ce qui n'est pas négligeable. Avec un tel constat, il parait paradoxal de s'attendre à ce que ces deux types d'instituteurs produisent des qualités identiques d'enseignements.

Notre motivation ici est de connaître la relation entre la qualité de la formation et les enseignements des instituteurs selon les professeurs des CAFOP (formés à l'ENS: École Normale Supérieure, structure de formation des enseignants du secondaire, du personnel d'encadrement et de supervision du Ministère de l'Éducation Nationale) et les conseillers pédagogiques des secteurs. Ainsi, la question fondamentale à laquelle il nous semble utile de répondre est la suivante :

Quels regards les professeurs des CAFOP et les conseillers pédagogiques de secteurs de l'enseignement primaire de Côte d'Ivoire portent-ils sur la formation et les enseignements dispensés par les instituteurs adjoints et les instituteurs ordinaires ?

L'objectif général visé est d'analyser les points de vue des professeurs du CAFOP et des conseillers pédagogiques de secteurs de l'enseignement primaire concernant la qualité des enseignements dispensés par les instituteurs adjoints et les instituteurs ordinaires de Côte d'Ivoire. Il s'agit dans ce sens :

-de recueillir les points de vue des professeurs des CAFOP et des conseillers pédagogiques des secteurs de l'enseignement primaire sur la qualité des enseignements dispensés par les instituteurs,

- d'identifier à partir de ces données, les problèmes réels que posent les enseignements des instituteurs.

L'hypothèse principale est centrée sur le fait que, bien qu'ayant eu à peu près la même qualité de formation pédagogique, la qualité des enseignements dispensés par les instituteurs adjoints est différente de celle des instituteurs ordinaires. Elle se décline ainsi :

Les professeurs des CAFOP et les conseillers pédagogiques du secteur de l'enseignement primaire estiment que la qualité des enseignements dispensés par les instituteurs adjoints est inférieure à celle des instituteurs ordinaires,

La qualité inférieure des enseignements dispensés par les instituteurs adjoints par rapport à ceux des instituteurs ordinaires est due au niveau inférieur de formation générale des instituteurs adjoints. 


\subsection{MÉTHODOLOGIE}

\subsection{LE CHAMP GÉOGRAPHIQUE DE LA RECHERCHE}

Trois villes nous ont intéressées. Ce sont Bouaké au centre, Katiola et Niakara au centre-nord de la Cote d'Ivoire. Nous avons choisi Bouaké parce que c'est la seule ville où il y a deux CAFOP. Dans cette ville nous nous sommes rendus dans les CAFOP 1 et 2 . Nous nous sommes également rendus à Katiola qui est la ville la plus proche ayant un CAFOP et nous avons terminé par Niakara, ville voisine. Ces deux premières villes n'ont pas d'autres particularités pour cette enquête, en dehors du fait qu'on y trouve les deux types de populations concernées par ladite enquête.

\subsection{POPULATION ET ÉCHANTILLONNAGE}

Cette étude a d'abord concerné les conseillers pédagogiques des secteurs de l'enseignement primaire. Nous avons visé ceux-là parce que lorsque les élèves-maîtres quittent les CAFOP et qu'ils sont affectés sur le terrain, ce sont les conseillers pédagogiques qui sont chargés de leur encadrement et de terminer leur formation. Ils ont suffisamment d'expérience en matière d'enseignement dans les écoles primaires et sont à même de juger du niveau de qualité de formation relatif à chaque instituteur lors de son stage de terrain.

La recherche a été réalisée sur 39 conseillers pédagogiques des secteurs des villes de Bouaké, de Katiola et de Niakara. Au total, 32 conseillers pédagogiques des secteurs ont pu répondre à nos questions, soit $82 \%$ des répondants.

L'étude a également concerné les professeurs des CAFOP. Nous nous sommes adressés à ces derniers car ce sont eux les personnes qui sont chargées de la formation pédagogique des élèves-maîtres qu'ils reçoivent. Ils savent quel genre de formation ils donnent aux deux types d'instituteurs.

Des 37 professeurs du CAFOP de Katiola, 24 ont répondu à nos questions. 20 professeurs du CAFOP 1 de Bouaké ont répondu sur 33 dont cet établissement compte. Au CAFOP 2 (ENI) de Bouaké, ce sont 22 réponses que nous avons eues sur 49 professeurs dont cet établissement compte. Au total, sur 119 professeurs appartenant à ces trois centres de formation pédagogique nous avons en tout 66 réponses, soit 55,5\%.

Commencée le 3 avril, cette enquête s'est terminée le 31 décembre de la même année. Le temps d'administration a été rallongé à cause des vacances scolaires. N'ayant pas pu avoir accès à suffisamment de personnes à enquêter, c'est à la rentrée scolaire suivante où nous avons parachevé l'enquête.

\subsection{INSTRUMENT D'OBSERVATION ET DE COLLECTE DES DONNÉES}

Le recueil des données s'est opéré à partir de deux questionnaires. Il s'agit d'un questionnaire adressé aux professeurs des CAFOP et d'un autre 
adressé aux conseillers pédagogiques des secteurs dans les inspections de l'enseignement préscolaire et primaire des trois villes indiquées. En ce qui concerne cette étude, avant de faire l'enquête proprement dite, nous avons d'abord fait une pré-enquête auprès d'une dizaine de professeurs des CAFOP. C'est à l'issue de cela que nous avons retenu les trois grandes fonctions sur lesquelles les professeurs des CAFOP insistent lors de la formation des élèvesmaîtres, à savoir : l'organisation, l'animation et l'évaluation. À cela, pour jauger la qualité des enseignements, s'ajoutent la préparation des cours, la maîtrise des contenus à enseigner, la volonté d'enseigner et la formation générale. Ce sont les mêmes dimensions qui ont été explorées dans les deux types de questionnaires.

\subsection{INSTRUMENT DE TRAITEMENT ET D'ANALYSE DES DONNÉES}

L'analyse descriptive ici a eu pour objectif de déterminer la répartition des modalités des différentes variables de la population d'étude. Une analyse de fréquence simple a été réalisée. Ensuite, essentiellement des tests de Chi 2 ont étés effectués pour déterminer la présence de liaisons entre les variables qualitatives de base. Les données ont étés traitées à l'aide du logiciel Excel.

\subsection{LES VARIABLES A L'ÉTUDE}

Pour vérifier la validité des hypothèses que nous avons définies plus haut, nous avons deux types de variables :

-La variable dépendante est l'appréciation que les répondants formulent par rapport à la qualité de l'enseignement. Ses indicateurs sont : bonne, assez bonne, passable, médiocre et mauvaise.

-La variable indépendante quant à elle, concerne le statut des répondants. Cette variable a deux modalités, à savoir les professeurs des CAFOP et les conseillers pédagogiques des secteurs. Les indicateurs de la variable indépendante sont l'organisation, l'animation, l'évaluation, la préparation des cours, la maîtrise des contenus à enseigner, la volonté d'enseigner et la formation générale.

\subsection{RÉSULTATS}

Les résultats font ressortir les appréciations de la qualité des enseignements dispensés par les instituteurs ordinaires et les instituteurs adjoints par les professeurs des CAFOP et les conseillers pédagogiques des secteurs de l'enseignement préscolaire et primaire. Nous présentons ces résultats en fonction des indicateurs du statut des répondants et des indicateurs sur la qualité des enseignements de ces derniers.

En premier point nous aurons l'appréciation générale sur la qualité des enseignements, puis en second point l'appréciation sur la formation générale 
que les élèves-maîtres ont eue dans les collèges et lycées avant de devenir des instituteurs.

\subsection{APPRÉCIATION GÉNÉRALE DE LA QUALITÉ DES ENSEIGNEMENTS}

Les enquêtés ont eu à se prononcer sur plusieurs critères : l'organisation, l'animation, l'évaluation, la préparation des cours, la maîtrise des contenus à enseigner et la volonté d'enseigner. Les réponses ont été recherchées tant chez les professeurs des CAFOP que chez les conseillers pédagogiques des secteurs.

\subsubsection{APPRÉCIATIONS SUR LA QUALITÉ DE L'ENSEIGNEMENT DES INSTITUTEURS ORDINAIRES}

Les points de vue des professeurs de CAFOP sur l'enseignement dispensé par les instituteurs ordinaires sont présentés dans le Tableau 1.

Tableau 1. Appréciation de la qualité de l'enseignement des instituteurs ordinaires par les

\begin{tabular}{|l|c|c|c|c|c|c|}
\multicolumn{7}{|c|}{ L'appréciation des répondants (en \%) } \\
\hline \multirow{2}{*}{$\begin{array}{l}\text { Professeurs } \\
\text { des CAFOP }\end{array}$} & Bonne & $\begin{array}{l}\text { Assez } \\
\text { bonne }\end{array}$ & Passable & Médiocre & Mauvaise & Total \\
\cline { 2 - 8 } & 69,7 & 16,7 & 7,6 & 4,5 & 1,5 & 100 \\
\hline Organisation & 62,1 & 21,2 & 12,1 & 3,1 & 1,5 & 100 \\
\hline Animation & 62,1 & 21,2 & 10,6 & 4,6 & 1,5 & 100 \\
\hline Évaluation & 59,1 & 19,7 & 15,2 & 3 & 3 & 100 \\
\hline $\begin{array}{l}\text { Préparation } \\
\text { de cours }\end{array}$ & 54,5 & 19,7 & 18,2 & 1,5 & 6,1 & 100 \\
\hline $\begin{array}{l}\text { Maîtrise des } \\
\text { contenus à } \\
\text { enseigner }\end{array}$ & 37,9 & 12,1 & 30,3 & 9,1 & 10,6 & 100 \\
\hline $\begin{array}{l}\text { Volonté } \\
\text { d'enseigner }\end{array}$ & 57,57 & 18,43 & 15,67 & 4,3 & 4,03 & 100 \\
\hline Total & & & & & & \\
\hline
\end{tabular}

La lecture de ce tableau nous permet de dire que dans l'ensemble, l'appréciation que les professeurs de CAFOP donnent sur les instituteurs ordinaires est bonne.

Le Tableau 2 nous présente les points de vue des conseillers pédagogiques des secteurs sur la qualité de l'enseignement des instituteurs ordinaires. 
Tableau 2. Appréciation de la qualité de l'enseignement des instituteurs ordinaires par les conseillers pédagogiques des secteurs

\begin{tabular}{|c|c|c|c|c|c|c|}
\hline \multirow{2}{*}{$\begin{array}{l}\text { Conseillers } \\
\text { pédagogiques } \\
\text { de secteurs }\end{array}$} & \multicolumn{6}{|c|}{ L'appréciation des répondants (en \%) } \\
\hline & Bonne & $\begin{array}{l}\text { Assez } \\
\text { bonne }\end{array}$ & Passable & Médiocre & Mauvaise & Total \\
\hline Organisation & 34,8 & 19,7 & 40,9 & 1,5 & 3,1 & 100 \\
\hline Animation & 15,2 & 33,3 & 36,4 & 10,6 & 4,5 & 100 \\
\hline Évaluation & 33,4 & 42,4 & 18,2 & 4,5 & 1,5 & 100 \\
\hline $\begin{array}{l}\text { Préparation de } \\
\text { cours }\end{array}$ & 25,7 & 28,8 & 34,9 & 3 & 7,6 & 100 \\
\hline $\begin{array}{lr}\text { Maîtrise } & \text { des } \\
\text { contenus } & \text { à } \\
\text { enseigner } & \\
\end{array}$ & 43,9 & 28,8 & 19,7 & 7,6 & 0 & 100 \\
\hline $\begin{array}{l}\text { Volonté } \\
\text { d'enseigner }\end{array}$ & 37,9 & 18,2 & 31,8 & 10,6 & 1,5 & 100 \\
\hline Total & 31,82 & 28,53 & 30,32 & 6,3 & 3,03 & 100 \\
\hline
\end{tabular}

Il ressort de ce tableau que l'appréciation que les conseillers pédagogiques de secteurs donnent sur les enseignements que dispensent les instituteurs ordinaires est assez bonne dans l'ensemble.

$\mathrm{Au}$ final, bien que les professeurs des CAFOP et les conseillers pédagogiques aient une bonne appréciation de la qualité de l'enseignement des instituteurs ordinaires, l'appréciation donnée par les professeurs des CAFOP est légèrement meilleure à celle des conseillers pédagogiques des secteurs.

\subsubsection{APPRÉCIATIONS SUR LA QUALITÉ DE L'ENSEIGNEMENT DES INSTITUTEURS ADJOINTS}

Les points de vue des conseillers pédagogiques des secteurs sur les enseignements des instituteurs adjoints sont présentés dans le Tableau 3.

Tableau 3. Appréciation de la qualité de l'enseignement des instituteurs adjoints par les conseillers pédagogiques de secteurs

\begin{tabular}{|c|c|c|c|c|c|c|}
\hline \multirow{2}{*}{$\begin{array}{l}\text { Conseillers } \\
\text { pédagogiques } \\
\text { de secteurs }\end{array}$} & \multicolumn{6}{|c|}{ L’appréciation des répondants (en \%) } \\
\hline & Bonne & $\begin{array}{l}\text { Assez } \\
\text { bonne }\end{array}$ & Passable & Médiocre & Mauvaise & Total \\
\hline Organisation & 33,4 & 22,7 & 40,9 & 1,5 & 1,5 & 100 \\
\hline Animation & 33,4 & 39,4 & 21,2 & 4,5 & 1,5 & 100 \\
\hline Evaluation & 28,8 & 45,5 & 19,7 & 6 & 0 & 100 \\
\hline $\begin{array}{l}\text { Préparation de } \\
\text { cours }\end{array}$ & 22,7 & 21,2 & 39,4 & 9,1 & 7,6 & 100 \\
\hline $\begin{array}{lr}\text { Maitrise } & \text { des } \\
\text { contenus } & \text { à } \\
\text { enseigner } & \\
\end{array}$ & 25,9 & 24,3 & 27,3 & 16,3 & 6,2 & 100 \\
\hline $\begin{array}{l}\text { Volonté } \\
\text { d'enseigner }\end{array}$ & 39,4 & 28,8 & 27,3 & 4,5 & 0 & 100 \\
\hline Total & 30,6 & 30,32 & 29,3 & 6,98 & 2,8 & 100 \\
\hline
\end{tabular}


Représentation graphique de l'appréciation de la qualité de l'enseignement des instituteurs adjoints par les conseillers pédagogiques de secteurs

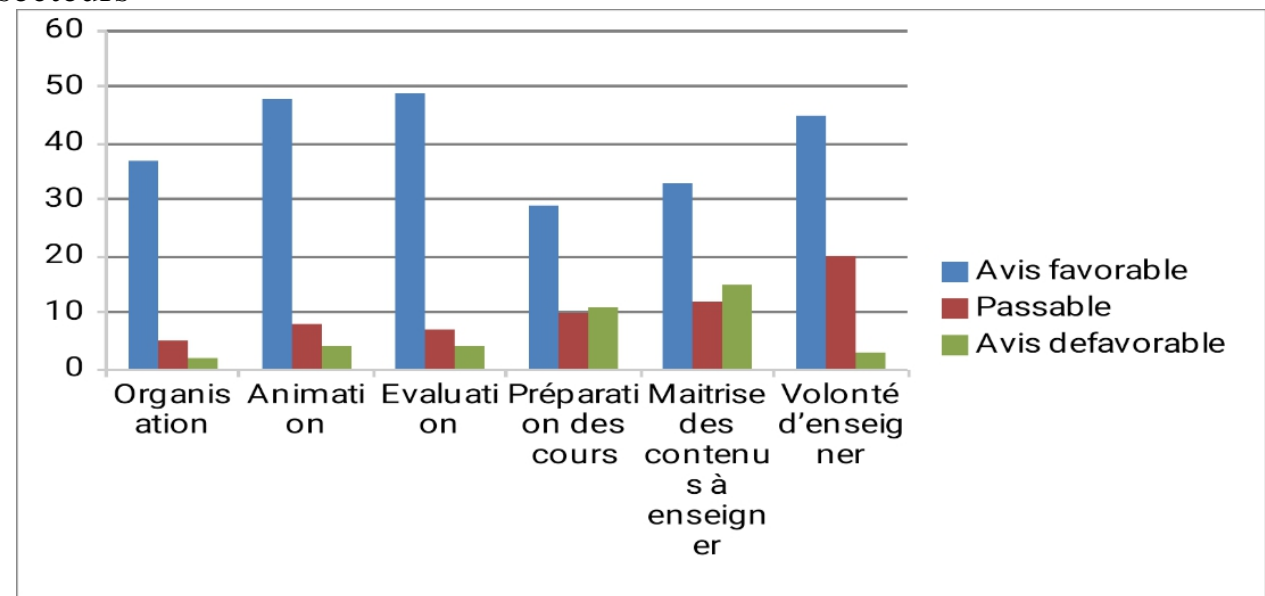

Figure 1. Source : Résultats de l'enquête avec les conseillers pédagogiques de secteurs primaire

À la vue du tableau ci-dessus, l'on constate que les conseillers pédagogiques des secteurs ont une assez bonne appréciation sur la qualité des enseignements que dispensent les instituteurs adjoints.

Quant à la qualité de l'enseignement des instituteurs adjoints, avec $X^{2}$ calculé $=43,516$ à 10 degrés de liberté et au seuil de $5 \%$, on retient que la qualité de l'enseignement des instituteurs adjoints dépend de l'appréciation des conseillers pédagogiques des secteurs ${ }^{1}$.

Le Tableau 4 nous présente les points de vue des professeurs des CAFOP sur les enseignements dispensés par les instituteurs adjoints.

Tableau 4. Appréciation de la qualité de l'enseignement des instituteurs adjoints par les professeurs des CAFOP

\begin{tabular}{|l|c|c|c|c|c|c|}
\hline \multirow{2}{*}{$\begin{array}{l}\text { Professeurs } \\
\text { des CAFOP }\end{array}$} & \multicolumn{6}{|c|}{ L'appréciation des répondants (en \%) } \\
\cline { 2 - 8 } & Bonne & $\begin{array}{l}\text { Assez } \\
\text { bonne }\end{array}$ & Passable & Médiocre & Mauvaise & Total \\
\hline Organisation & 63,6 & 22,7 & 4,5 & 3,1 & 6,1 & 100 \\
\hline Animation & 57,6 & 24,2 & 9,1 & 0 & 9,1 & 100 \\
\hline Évaluation & 60,6 & 21,2 & 12,1 & 1,5 & 4,6 & 100 \\
\hline $\begin{array}{l}\text { Préparation } \\
\text { de cours }\end{array}$ & 50 & 25,8 & 18,2 & 3 & 3 & 100 \\
\hline $\begin{array}{l}\text { Maîtrise des } \\
\text { contenus à } \\
\text { enseigner }\end{array}$ & 33,3 & 13,6 & 12,1 & 16,7 & 24,3 & 100 \\
\hline $\begin{array}{l}\text { Volonté } \\
\text { d'enseigner }\end{array}$ & 37,9 & 21,2 & 9,1 & 18,2 & 13,6 & 100 \\
\hline Total & 50,5 & 21,45 & 10,85 & 7,08 & 10,12 & 100 \\
\hline
\end{tabular}


Représentation graphique de l'appréciation de la qualité de l'enseignement des instituteurs adjoints par les professeurs de CAFOP

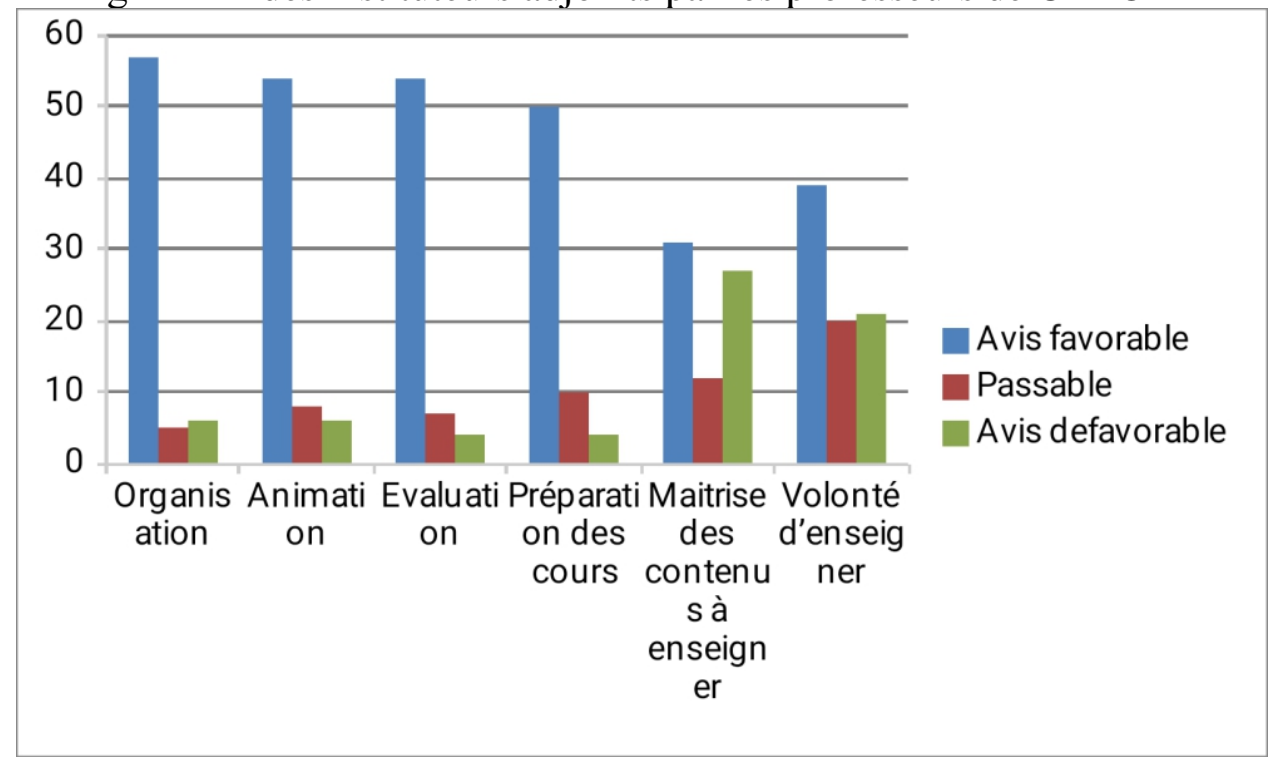

Figure 2. Source : Résultats de l'enquête avec les professeurs de CAFOP

L'analyse du Tableau 4 ci-dessus nous indique que c'est un assez bon enseignement celui que les instituteurs adjoints dispensent dans leurs classes selon les professeurs des CAFOP.

Quant à la qualité de l'enseignement des instituteurs adjoints, avec $\mathrm{X}^{2}$ calculé $=69,152$ à 10 degrés de liberté et au seuil de $5 \%$, on retient que la qualité de l'enseignement des instituteurs adjoints dépend de l'appréciation des professeurs des CAFOP.

Nous déduisons des Tableaux 3 et 4 que l'appréciation que les professeurs des CAFOP et les conseillers pédagogiques des secteurs donnent sur la qualité de l'enseignement des instituteurs adjoints est sensiblement la même.

Cette configuration des répondants est-elle influencée de façon significative quand ce sont les professeurs des CAFOP ou les conseillers pédagogiques des secteurs? En référence aux les résultats des tests de Khideux sur l'appréciation de la qualité des enseignements des instituteurs adjoints du côté des conseillers pédagogiques et des professeurs des CAFOP dépendent de leurs appréciations, ce qui veut dire que la bonne qualité de l'enseignement des instituteurs ordinaires n'est pas la même chez les professeurs des CAFOP et les conseillers pédagogiques des secteurs.

Autrement dit, la qualité d'enseignement des instituteurs adjoints ne varie pas en fonction des professeurs des CAFOP ou des conseillers pédagogiques des secteurs. 
2.2 APPRÉCIATION GÉNÉRALE DE LA QUALITÉ DE LA FORMATION GÉNÉRALE RECUE PAR INSTITUTEURS

Les enquêtés ont eu à se prononcer sur la qualité de la formation que les élèves-maîtres ont eu avant d'entrer dans les CAFOP et celle qu'ils ont en arrivant sur le terrain pour tenir une classe.

\subsubsection{APPRÉCIATIONS DONNÉES PAR LES PROFESSEURS DES CAFOP SUR LA QUALITÉ DE LA FORMATION GÉNÉRALE RECUE PAR INSTITUTEURS}

Les professeurs des CAFOP estiment que du nombre des instituteurs ordinaires recrutés avec le BAC qu'ils reçoivent, $60 \%$ d'entre eux ont un bon niveau, contre $37 \%$ chez les instituteurs adjoints recrutés avec le BEPC.

C'est un niveau assez bon qui est attribué aux instituteurs ordinaires par les professeurs des CAFOP concernant la formation générale reçue par les instituteurs ordinaires. Ces mêmes professeurs trouvent que le niveau des instituteurs adjoints est nettement en dessous de la moyenne.

\subsubsection{APPRÉCIATIONS DONNÉES PAR LES CONSEILLERS PEDAGOGIQUES DE SECTEURS SUR LA QUALITÉ DE LA FORMATION GÉNÉRALE RECUE PAR INSTITUTEURS}

Pour les conseillers pédagogiques des secteurs, des instituteurs ordinaires qu'ils reçoivent, $72 \%$ d'entre eux ont un bon niveau de formation acquis dans les collèges et lycées, tandis que $58 \%$ des instituteurs ordinaires recrutés à partir du BEPC ont un bon niveau dans l'ensemble.

La variable appréciation de la qualité des enseignements est influencée par le niveau de formation générale des instituteurs avec $X^{2}=0,049$ à 3 degrés de liberté, au seuil de 5\%. L'examen des chiffres montre que ce soit pour les instituteurs ordinaires ou adjoints, les conseillers pédagogiques estiment qu'ils ont tous deux un niveau supérieur à la moyenne. Le niveau de formation générale des instituteurs ordinaires est nettement supérieur à celui des instituteurs adjoints.

Tels sont là exposés, les résultats de cette étude. Que faut-il en penser ?

\subsection{DISCUSSION}

L'objectif général de ce travail, faut-il le rappeler, est d'analyser les points de vue des professeurs des CAFOP et des conseillers pédagogiques de secteurs du primaire concernant la qualité des enseignements dispensés par les instituteurs adjoints et les instituteurs ordinaires de Côte d'Ivoire.

Celui-ci a ensuite été décliné en deux objectifs spécifiques, à savoir : 
1. Recueillir les points de vue des professeurs des CAFOP et des conseillers pédagogiques des secteurs sur la qualité des enseignements dispensés par les instituteurs ;

2. Identifier à partir de ces données, les problèmes réels que posent les enseignements des instituteurs.

L'examen des résultats exposés nous permet de noter que ces objectifs ont été atteints. En effet, l'enquête que nous avons conduite a permis de recenser les points de vue des conseillers pédagogiques des secteurs et des professeurs des CAFOP sur la qualité de la formation et celle des enseignements dispensés par les instituteurs ordinaires et adjoints. Ainsi, les deux catégories de personnes interrogées ont mis en exergue les principales difficultés rencontrées et ont ensuite émis des pistes de solutions.

En rapport avec ces objectifs, des hypothèses avaient été formulées et il s'agissait de les vérifier sur le terrain.

L'hypothèse principale est la suivante : bien qu'ayant eu à peu près la même qualité de formation pédagogique, la qualité des enseignements dispensés par les instituteurs adjoints est différente de celle des instituteurs ordinaires.

À partir de cette hypothèse principale, des hypothèses secondaires ont été formulées, à savoir :

- Les professeurs des CAFOP et les conseillers pédagogiques de secteurs estiment que la qualité des enseignements dispensés par les instituteurs adjoints est inférieure à celle des instituteurs ordinaires ;

- La qualité inférieure des enseignements dispensés par les instituteurs adjoints par rapport à ceux des instituteurs ordinaires est due au niveau inférieur de formation générale des instituteurs adjoints.

Ces hypothèses sont-elles vérifiées sur le terrain?

\subsection{LA PREMIÈRE HYPOTHÈSE}

Les données recueillies sur le terrain, analysées, nous permettent d'affirmer d'abord que la qualité de la formation reçue par les instituteurs adjoints et les instituteurs ordinaires est quasiment la même. Ce sont les mêmes professeurs qui enseignent ces deux catégories d'instituteurs et la formation se fait dans les mêmes conditions. Quel que soit le sexe, c'est dans une large majorité que la confirmation de la qualité similaire de formation a été faite.

À propos de la qualité des enseignements dispensés par les instituteurs ordinaires et les instituteurs adjoints, la majorité des enquêtés, conseillers pédagogiques et professeurs des CAFOP, estiment que la qualité des enseignements dispensés par les instituteurs ordinaires est supérieure à celle dispensée par les instituteurs adjoints. Les nombreuses lacunes tant dans le 
langage écrit qu'oral en sont pour quelque chose. La variable sexe n'a pas d'incidence significative sur les points de vue exprimés par les différentes personnes enquêtées.

L'on est passé de la formation des instituteurs avec le BAC, à celle de la formation avec le BEPC. Tout changement, surtout en éducation, suscite des questionnements et même des inquiétudes. Lenoir, Larose et Lessard (2005) indiquent que souvent les fondements de la reforme sont loin d'être toujours très clairs et cohérents. Il ne faut certes pas être réfractaire au changement, mais il faut, comme ils le disent, que les motivations de ce changement soient cohérentes. Malgré le changement, la qualité de la formation dispensée dans les CAFOP est quasiment restée identique.

\subsection{LA DEUXIÈME HYPOTHÈSE}

Les prestations de ces deux catégories d'enseignants devaient en principe être identiques. Ce n'est pas ce qu'il nous a été donné à constater avec les résultats. En effet, la majorité des enquêtés (professeurs et conseillers) ont reconnu que les enseignements donnés par les instituteurs adjoints sont de qualité inférieure à ceux des instituteurs ordinaires. La réponse est donc en dehors de la formation reçue dans les CAFOP.

Les enquêtés ont reconnu également que dans une promotion donnée d'instituteurs adjoints, ceux qui n'ont pas un bon niveau de formation générale atteignent $19 \%$, tandis que pour les instituteurs ordinaires, le pourcentage est de $6 \%$.

Dans une grande majorité aussi, les professeurs des CAFOP $(85,7 \%)$ et les conseillers pédagogiques de secteurs (72\%) ont reconnu que les instituteurs adjoints présentaient plus de lacunes dans la conduite de leurs leçons, tant à l'oral qu'à l'écrit, que les instituteurs ordinaires, ceci à cause de la formation générale qu'ils ont reçu avant de se rendre à l'enseignement.

Enfin, les deux catégories d'enquêtés ont retenu le niveau de formation générale comme la principale raison de la différence de qualité des enseignements observée chez les instituteurs adjoints par rapport aux instituteurs ordinaires.

Que faut-il retenir de là ? Nous retenons alors que la formation dispensée dans l'enseignement général est très importante car elle a un impact sur les enseignements dans nos écoles. «L'art de l'éducation est comme l'art médical : un art qui ne peut être pratiqué sans «dons » spéciaux mais un art supposant des connaissances exactes et expérimentales, relatives aux êtres humains sur lesquels il s'exerce. Ces connaissances ne sont pas anatomiques et physiologiques, comme celles du médecin, mais psychologiques.» (Piaget, 1971, pp. 88, 89). Tout en concordance avec Piaget, nous sommes d'avis que l'art de l'éducation doit être maitrisé par ses praticiens. Pour cela, il faut une bonne formation. Cette dernière commence depuis l'école primaire et continue 
dans les collèges et les lycées. La formation générale acquise dans ces lieux est primordiale pour mieux acquérir, dans les CAFOP, les connaissances psychologiques sur les élèves. Plus la formation de nos élèves dans les collèges et les lycées est de bonne qualité, plus les enseignements dispensés dans nos écoles primaires le seront aussi. (Meirieu, 2009, p.134) souligne : « On ne peut enseigner qu'en s'appuyant sur ses acquis ... rien ne peut être acquis sans que l'apprenant l'articule sur ce qu'il sait déjà.». Pour relever le niveau de l'enseignement, il faut relever le niveau de recrutement des enseignants car plus le niveau auquel on a recruté l'enseignant est élevé en comparaison avec celui de l'élève qu'il doit enseigner, plus il donnera de meilleurs résultats.

La motivation qui anime les enseignants est difficile à appréhender parce qu'elle constitue un concept que l'on ne peut ni toucher, ni voir, ni observer directement ou mesurer (Wlodwski, 1999). Concernant la volonté accrue chez les instituteurs adjoints, la raison principale qui explique cela est que les instituteurs adjoints ont encore du chemin à parcourir dans l'enseignement primaire. Ils doivent passer un examen écrit et pédagogique pour devenir des instituteurs ordinaires. Un apprentissage réussi est un apprentissage qui permet des applications, des réinvestissements et même des transferts (Develay, 1996). Dans cette recherche d'ascension professionnelle, ils fournissent beaucoup d'efforts lors de leurs enseignements mais le faible niveau de formation générale qu'ils ont reçu dans les lycées et collèges constitue un frein empêchant d'accéder à de meilleurs enseignements.

La reforme éducative est souvent employée pour désigner l'introduction d'un changement dans les structures, les contenus ou les pratiques éducatives (Pastiaux, 2006). Plusieurs mécanismes s'avèrent importants pour mettre en œuvre une reforme éducative. Le but des reformes éducatives est d'améliorer l'existant ; en d'autres termes, d'atteindre la qualité et la pertinence dans un système éducatif donné. La formation et la profession enseignante sont souvent au cœur du débat dans les reformes éducatives. La reforme ici a consisté à passer du recrutement des instituteurs du BAC au BEPC.

Le succès de la reforme repose en grande partie sur la quantité et la qualité de la formation qui sera donnée au corps enseignant (Lasnier, 2000). Concernant le volet quantité, on l'a relevé. Le nombre d'années de formation est essentiel. Une seule année de formation est insuffisante; le minimum raisonnable serait de 2 années. Quant au volet qualité, il est tout aussi important. L'insuffisance de formation générale que les élèves ont eue quand ils étaient dans les collèges et lycées doit être comblée quand ils sont en formation dans les CAFOP. À défaut, il faut qu'ils soient bien formés, avec comme niveau minimum le BAC avant d'entrer dans les centres de formation pédagogique. La bonne formation pédagogique est nécessaire pour avoir un enseignement de qualité (Nkeck, 2015, p.138). 
Séri (1980, p.33) souligne : « des milliers de tonnes de « rapports scientifiques » ont été produits qui tendaient à faire croire que trois mots seraient vaincus : les déperditions scolaires, le caractère inadéquat du contenu des programmes, les coûts excessifs». À cette bataille, aucune compétence scientifique ivoirienne n'a été associée au cours des années 1967-1968, 19691970. Et pourtant, ces études scientifiques engageaient lourdement l'avenir du pays !». Certes, il parlait de la télévision éducative à l'école primaire, mais ce même secteur, s'il bénéficiait aujourd'hui d'études stratégiques concernant la formation, nous sommes convaincus que nous ne serions pas arrivés à un tel changement concernant le diplôme à partir duquel l'on peut accéder à l'enseignement dans l'école primaire. Le volet financier nous semble léger pour prendre une telle décision; l'avis des compétences scientifiques gagnerait à être pris en compte dans ce genre de situations qui peuvent impacter grandement l'avenir du pays en général et du système éducatif en particulier.

\subsection{CONCLUSION}

Nous étions partis à l'entame de notre recherche, du fait que la qualité de la formation donnée dans les CAFOP aux deux catégories d'instituteurs qui sont les ordinaires et les adjoints était sensiblement la même. Nos investigations ont corroboré cela et selon les conseillers pédagogiques, et surtout les professeurs des CAFOP, cette formation est d'assez bonne qualité quand bien même des propositions ont été faites pour son amélioration.

$\mathrm{Au}$ terme de cette étude, nous retenons que les enseignements dispensés par les instituteurs adjoints sont de qualité inférieure à ceux des instituteurs ordinaires. Ceci s'explique par la formation générale des instituteurs adjoints qui est inférieure à celle des instituteurs ordinaires.

Etant donné que la qualité des enseignements dispensés par les instituteurs ordinaires est nettement supérieure à celle des instituteurs adjoints, il va sans dire que c'est l'existence de la première catégorie d'instituteurs qu'il faut privilégier. L'insuffisance de ressources financières ne nous semble pas une raison suffisante pour faire le choix de l'option du recrutement des instituteurs adjoints. Tandis que dans des pays développés, tels que la France et le Canada, c'est après des diplômes de l'enseignement supérieur que l'on vient enseigner à l'école primaire, l'on comprend mal qu'ici, dans ce pays qui aspire au développement, ce soit la voie contraire qui est empruntée. Après le $\mathrm{BAC}$, c'est maintenant à partir du BEPC que l'on recrute pour enseigner dans les écoles primaires sous nos cieux; c'est une régression. Nous disons qu'à défaut de recruter à partir des études universitaires, il faut tout au moins retourner aux recrutements à partir du $\mathrm{BAC}$ et en plus faire passer le temps de la formation dans les CAFOP d'une année à deux années au minimum. Si toutefois l'État tient au recrutement à partir du BEPC pour enseigner, il 
faudrait que ce soit pour enseigner au préscolaire ou tout au plus jusqu'à la classe de CP2. Le niveau de langue et la formation initiale acquis ne pourront pas dans un tel cas être un frein aux enseignements de qualité que ces enseignants auraient à dispenser. Cette réforme du système ne serait pas une raison visant à dire que l'enseignement préscolaire n'est pas important mais que l'apport des enseignants titulaires du BEPC serait meilleure au préscolaire qu'au primaire.

Autant le désastre pédagogique des années 70 occasionné par l'implantation de la télévision éducative aurait pu être évité à temps si la Côte d'Ivoire post coloniale s'était débarrassée de son complexe d'infériorité qui veut que tout ce qui vient de l'extérieur soit considéré comme excellent, autant l'on peut éviter actuellement le désastre qui se met en place progressivement avec le recrutement massif d'instituteurs adjoints au détriment d'instituteurs ordinaires simplement parce qu'un groupe de décideurs non représentatifs et n'ayant pas suffisamment apprécié les conséquences de tels actes en a décidé ainsi.

La qualité de l'enseignement dispensé est en grande partie l'œuvre de ceux qui la dispensent, il convient donc qu'un accent particulier soit mis sur leur recrutement et leur formation pour un système éducatif plus performant.

Des études plus approfondies permettront certainement de mieux faire comprendre l'importance d'un capital humain de qualité pour l'enseignement primaire et, de façon plus générale, pour l'éducation.

\section{References:}

1. Bloom, B. (1969). Taxonomie des objectifs pédagogiques, Tome 1: domaine cognitif. Montréal: Edition Nouvelle.

2. Banque Mondiale (2003). Rapport mondial sur le développement. New-York: Oxford University Press.

3. Develay, M. (1996). Peut-on former les enseignants ? Paris : ESF.

4. Faure, B. (2008). «Décomplexer les SIC-Assumer le couple info-com et l'interdisciplinarité», actes du XVIe congrès SPSIC. Disponible sur http:/www.sfsic.org-2008/spip.php ? article140.

5. Koffik, A. (2012). Motivation et difficultés de stagiaires en formation d'éducateurs à l'ENS. Revue African Developpement Issues (AEDI), $\mathrm{N}^{\circ} 4,61-85$.

6. Lasnier, F. (2000). Réussir la Formation par Compétences. Québec : Edition Guerin.

7. Lenoir, Y., Larose, F., \& Lessard, C. (Dir) (2005). Le curriculum de l'enseignement primaire : regards critiques sur les fondements et les lignes directrices sherbrooke: CRP.

8. Meirieu, P. (2009). Apprendre ... oui, mais comment ? Paris : ESF. $193 \mathrm{p}$. 
9. Nkeck, B.R.S. (2015). Formation professionnelle et pratique enseignante de l'instituteur débutant. Revue JERA / RARE N ${ }^{\circ}$ 7. 125143.

10. Pastiaux, G. (2006). La pédagogie, 3è éd. Paris : Nathan.

11. Piaget, J. (1971). Où va l'éducation ? Comprendre, c'est inventer. Paris : Denoël, / Gonthier.

12. Seri, D. (1980). La place de l'art musical dans l'éducation ivoirienne. Annales de l'Université d'Abidjan, Serie F tome IX, 9-34.

13. UNESCO (2002). Education for all: is the world on Track ? EFA Global monitoring report 2002. Paris: UNESCO.

14. Wlodkowski, R.J. (1999). Enhancing Adult Motivation to learn, San Francisco, Jossey- Bass Publishers.

15. Woodhouse, D. (1999). Qualité et assurance-qualité, Dans OCDE, Qualité et internalisation de l'enseignement supérieur. 